\section{Opportunistic infections in a renal transplant recipient}

\author{
Vijaya V. Mysorekar, \\ Mahesh Eshwarappa, Umesh Lingaraj \\ Departments of Pathology and \\ Nephrology, M.S. Ramaiah Medical \\ College and Hospital, Bangalore, India
}

\begin{abstract}
With the present progress in transplantation procedures, there is an improvement in patient and allograft survival. However, the immunosuppression necessary to sustain the allograft predisposes these transplant recipients to infection, which is now a significant cause of morbidity and mortality. We describe a case of a 30-year-old renal transplant recipient with two opportunistic infections, namely, primary cutaneous aspergillosis and intestinal tuberculosis, with terminal enterococcal pleuritis and peritonitis. Control of the degree of immunosuppression, and prompt recognition and treatment of infection are vital for successful organ transplantation.
\end{abstract}

\section{Introduction}

Solid organ transplantation is becoming increasingly common in the treatment of endstage organ failure. The two crucial factors for successful organ transplantation are: i) better control of rejection by administration of immunosuppressant therapy, and ii) better prevention and treatment of infection which transplant patients are more predisposed to, on account of the immunosuppressioninduced compromised cell-mediated immunity. Up to $15 \%$ of renal transplant recipients die of opportunistic infections. ${ }^{1}$ Infections are most common during the first 6 months post-transplant. ${ }^{2,3}$ Infections may be exogenous in origin due to environmental exposure to infective agents, or they may occur as a result of invasion by endogenous opportunistic agents (bacteria, viruses or fungi). We report two opportunistic infections, namely, primary cutaneous aspergillosis and intestinal tuberculosis, with terminal enterococcal pleuritis and peritonitis, in a renal transplant recipient.

\section{Case Report}

A 30 year old male patient who was a case of chronic glomerulonephritis and had developed end-stage renal disease, had received a renal transplant 10 months back. He was on immunosuppressant therapy (tacrolimus 4 $\mathrm{mg} /$ day, mycophenolate mofetyl $1.5 \mathrm{gm} /$ day and steroid prednisolone $7.5 \mathrm{mg} /$ day). He presented with a 6-month history of a mass in the right sole, discharging pus. Prior to this, he had a long-standing wound of 2 months duration at the same location, close to the heel, which he attributed to pressure from his shoe. The wound was not present prior to the transplantation. On examination, the mass was tender, firm, indurated, $4 \times 5 \mathrm{~cm}$ in size, situated subcutaneously. The overlying skin was dark brown. His haematological and biochemical parameters were normal.

The mass was excised along with the overlying elliptical part of skin and the deeper subcutaneous tissue. Macroscopic examination of the excised specimen showed multiple sinuses 0.2 to $0.4 \mathrm{~cm}$ in size on the skin surface, draining whitish pus. The cut surface showed necrotic material. On microscopic examination, ulcerated skin was seen. In the keratin layer of the epidermis, and in the dermis, there were numerous fungal hyphae which appeared dark brown, septate, and showed acute-angle branching (Figures 1 and 2). The fungus was surrounded by neutrophilic microabscesses. Dense inflammatory cell infiltrates were extending up to the subcutaneous fat. Fungal culture from the tissue isolated Aspergillus niger. A thorough search did not reveal any focus of Aspergillus infection anywhere else in the body. The patient tested seronegative for human immunodeficiency virus (HIV) infection. He was started on antifungal therapy, viz. oral voriconazole $200 \mathrm{mg} /$ day. The dosage of immunosuppressant tacrolimus was reduced.

One month later, during a follow-up visit for his renal status, the patient showed signs of graft dysfunction, his serum creatinine being $2.5 \mathrm{mg} / \mathrm{dL}$ (normal range $0.6-1.2 \mathrm{mg} / \mathrm{dL}$ ). A renal biopsy was done. It showed normal glomeruli and blood vessels. There was evidence of mild tubulitis (1-4 mononuclear cells/tubular cross section). The interstitium showed oedema and a moderate degree of inflammation with infiltration by mainly lymphocytes admixed with macrophages, affecting about $10 \%$ of the parenchyma. The findings were categorized as borderline changes, suspicious of acute rejection (Banff classification $2007^{4}$ ). This necessitated anti-rejection therapy with pulse dose methylprednisolone intravenously for 3 days, followed by oral prednisolone. The patient discontinued the antifungal therapy on his own. He did not visit the concerned clinician for follow-up, and hence a repeat fungal culture or biopsy from the surgical site was not done.

Six weeks later, the patient complained of pain in abdomen of 5 days duration, and vomiting since 2 days. He was diagnosed to have
Correspondence: Dr. Vijaya V. Mysorekar, 89 A.G.'s office colony, $5^{\text {th }}$ main, 6 th cross, New BEL Road, Bangalore - 560054. India.

Tel :91.080.23600395 - Fax: 91.080.23606213.

E-mail: vijayamysorekar@yahoo.com

Key words: Aspergillosis, tuberculosis, opportunistic infections, renal transplantation.

Acknowledgement: the authors are grateful to Dr. Saraswathi G. Rao, Principal and Dean, M. S. Ramaiah Medical College, Bangalore, India, for her kind support for publishing this paper.

Contributions: VVM, histopathology reporting, article drafting; ME, UL, patient treating, article critically reviewing, final version approving.

Conflict of interest: the authors report no conflicts of interest.

Received for publication: 2 October 2011.

Revision received: 20 November 2011.

Accepted for publication: 27 December 2011.

This work is licensed under a Creative Commons Attribution NonCommercial 3.0 License (CC BYNC 3.0).

(C) Copyright V.V. Mysorekar et al., 2012 Licensee PAGEPress, Italy

Infectious Disease Reports 2012; 4:e8 doi:10.4081/idr.2012.e8

peritonitis secondary to hollow viscus (ileal) perforation. A haemogram done revealed haemoglobin $7.0 \mathrm{gm} / \mathrm{dL}$ (normal range 13.0$18.0 \mathrm{gm} / \mathrm{dL}$ ), haematocrit $20.4 \%$ (normal range 40-54\%), total leukocyte count 10,400 cells/ $\mu \mathrm{L}$ with $74 \%$ neutrophils and $22 \%$ lymphocytes (normal range 4,000-11,000 cells/ $\mathrm{LL}$ with 40 $75 \%$ neutrophils and $20-50 \%$ lymphocytes), and platelet count 85,000 cells/ $\mu \mathrm{L}$ (normal range $1,50,000-4,00,000 \mathrm{cells} / \mu \mathrm{L})$. The patient underwent laparotomy. A segment of perforated ileum was resected and subjected to histopathological examination. Grossly, the segment of ileum measured $7 \mathrm{~cm}$ in length. It showed a central perforation measuring $1 \mathrm{~cm}$ in diameter, surrounded by shaggy mucosa. Microscopically, the intestinal mucosa showed large areas of ulceration (Figure 3A). There was dense transmural infiltration by neutrophils and lymphocytes (Figures 3A and 3B). An occasional ill-formed epithelioid granuloma was seen. Staining for acid fast bacilli revealed strong positivity, confirming the presence of Mycobacterium tuberculosis (Figure 4). A diagnosis of ileal tuberculosis with perforation, was made.

Further investigation by sputum smear staining revealed acid fast bacilli, indicating that the patient also had pulmonary tuberculosis. He had pleural effusion and mild ascites. Culture of the pleural and peritoneal fluids revealed enterococcus; hence enterococcal 
pleuritis and peritonitis was diagnosed. The patient was started on antituberculous RIPE therapy (with rifampicin $600 \mathrm{mg} /$ day, isoniazid $300 \mathrm{mg} /$ day, pyrazinamide $1500 \mathrm{mg} /$ day and ethambutol $1500 \mathrm{mg} /$ day), along with intravenous antibiotics. However, six days later, he developed signs of septic shock and expired.

\section{Discussion}

Primary cutaneous aspergillosis in renal transplant patients is extremely rare $^{5}$ as compared to pulmonary aspergillosis. It occurs due to the immunocompromised state, and usually involves sites of skin injury, such as intravenous catheter sites, sites of traumatic inoculation, under adhesive dressings, burns or surgical wounds. ${ }^{5,6}$ In a study by Grossi et al.. ${ }^{7}$ Aspergillus niger infection accounted for $3.8 \%$ of all invasive fungal infections in thoracic organ transplant recipients. In a review of reports of cutaneous aspergillosis, van Burick ${ }^{6}$ found that Aspergillus niger species was causative in $6 \%$ of cases involving non-HIVinfected populations. Thomas et al. ${ }^{8}$ claim to report the first case of primary cutaneous

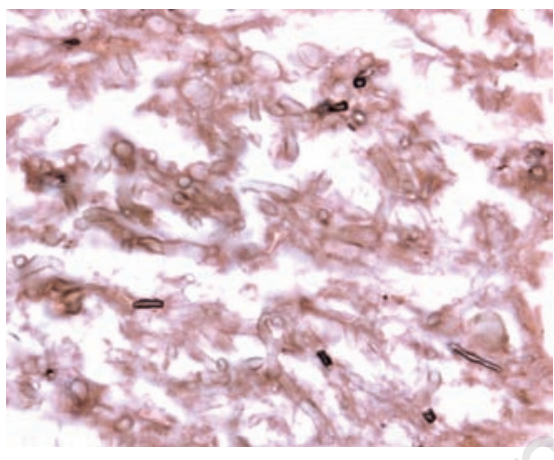

Figure 1. Photomicrograph showing dark brown, septate fungal hyphae with acuteangle branching (Haematoxylin and Eosin $x$ 1000).

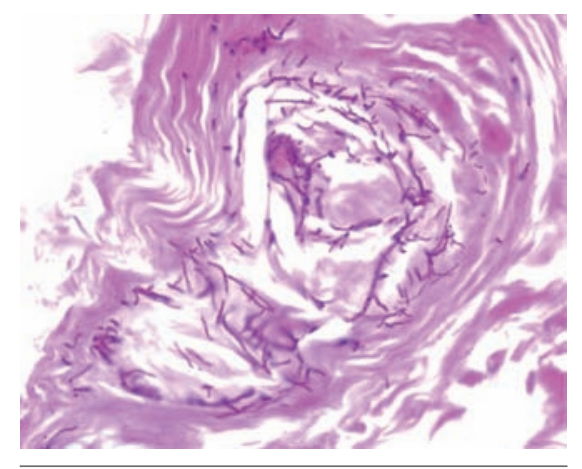

Figure 2. Photomicrograph showing fungal hyphae in the keratin layer of the epidermis, highlighted by periodic acid Schiff staining (magenta colour) (Periodic acid Schiff $x$ 400). aspergillosis caused by Aspergillus niger, in a surgical wound in a renal transplant recipient. In the present case, Aspergillus niger colonisation occurred in a long-standing pressure sore which developed post-transplant, most likely to have been caused by the patient's footwear. Primary cutaneous aspergillosis in solid-organ transplant recipients generally occurs in the setting of a normal neutrophil count, ${ }^{6}$ as in the present case. In all these patients, efforts should be made to search for evidence of spread to an extracutaneous site, such as the lung. Our patient had no evidence of extracutaneous aspergillosis.

Organ transplant recipients are at a high risk for development of Mycobacterium tuberculosis infection. The prevalence of tuberculosis in these patients, in various studies, has ranged from $0.26-10 \%$, being higher in developing countries. ${ }^{1,9-12}$ The mean duration of time between transplantation and the occurrence of tuberculosis in various reports has ranged from 1 to 21 months., ${ }^{10-12}$ Mycobacterium tuberculosis infection in renal transplant recipients usually occurs due to reactivation of latent tuberculous lesions. ${ }^{1}$ Our patient had not undergone testing for latent tuberculous infection prior to transplantation. There is a

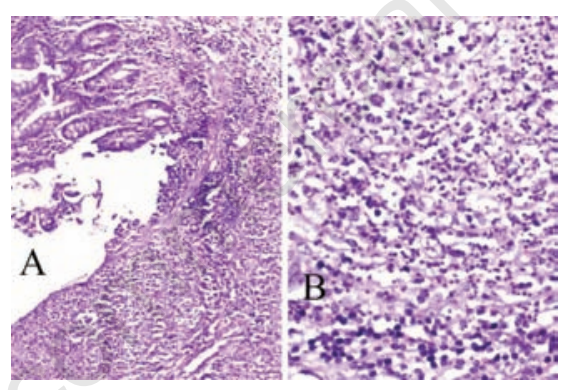

Figure 3. A) Photomicrograph showing ulcerated intestinal mucosa with dense inflammatory cell infiltration (Haematoxylin and Eosin x 100); B) dense infiltration by neutrophils and lymphocytes, in the intestinal wall (Haematoxylin and Eosin $x$ 400).

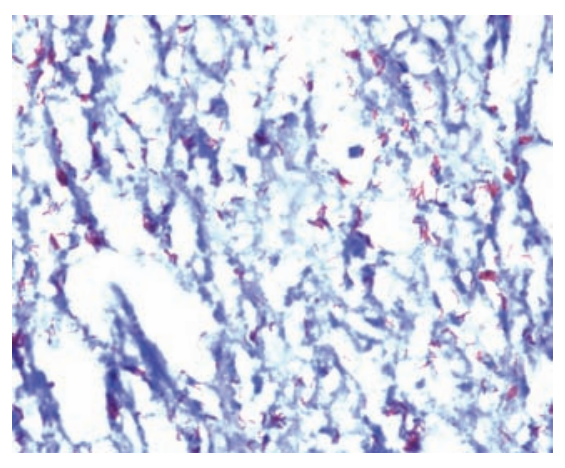

Figure 4. Photomicrograph showing strong positivity for acid fast bacilli (ZiehlNeelsen stain $x$ 1000). high rate of extrapulmonary tuberculosis in renal transplant recipients. ${ }^{1,11}$ The symptoms of infection are often attenuated or atypical, leading to delayed diagnosis. Hence, many of these patients already have disseminated tuberculosis at the time of presentation. Due to the high frequency of anergy in immunosuppressed patients, a false-negative result is often obtained with the Mantoux test, further adding to the difficulty in diagnosis.

The present case emphasizes that opportunistic infections are an important cause of morbidity and mortality among renal transplant recipients. The use of newer immunosuppressive agents such as tacrolimus and mycophenolate mofetyl, antirejection therapy with steroids, the general state of malnutrition in transplant recipients, the endemic nature of certain infections, lack of proper hygiene, and lack of protective measures against infection, particularly in the hospital environment are factors contributing to the high incidence of opportunistic infections in renal transplant recipients. ${ }^{1}$ Regular monitoring of the level of immunosuppression is critical. Early suspicion and an aggressive diagnostic approach are important for timely diagnosis and treatment of these infections. However, there are unfortunate situations, as in the present case, where the clinician has to deal with multiple complications, and it becomes very difficult to strike a balance between immunosuppression and control of infection. The use of prophylactic antimicrobial agents and vaccinations may be helpful in certain cases. ${ }^{1}$

\section{References}

1. Vinod PB, Sharma RK. Opportunistic infections (non-cytomegalovirus) in live related renal transplant recipients. Indian J Urol 2009;25:161-8.

2. Locnen SR, Pena AC, Abrihan-Arce Y, Cerezo CU. Infectious disease complications of renal transplantation at the University of the East Ramon Magsaysay Memorial Medical Center (UERMMMC). Phil J Microbiol Infect Dis 2000;29:119-22.

3. Fishman JA, Rubin RH. Infection in organtransplant recipients. New Eng J Med 1998;338:1741-51.

4. Solez K, Colvin RB, Racusen LC, et al. Banff 07 classification of renal allograft pathology: updates and future directions. Am J Transplant 2008;8:753-60.

5. Mallat SG, Aoun M, Moussalli A, et al. Cutaneous aspergillosis in a renal transplant recipient. J Med Liban 2004;52:111-4.

6. van Burik JA, Colven R, Spach DH. Cutaneous aspergillosis. J Clin Microbiol 1998;36:3115-21.

7. Grossi P, Farina C, Fiocchi R, Dalla 
Gasperina D. Prevalence and outcome of invasive fungal infections in 1,963 thoracic organ transplant recipients: a multicenter retrospective study. Italian study group of fungal infections in thoracic organ transplant recipients. Transplantation 2000;70:112-6.

8. Thomas LM, Rand HK, Miller JL, Boyd AS. Primary cutaneous aspergillosis in a patient with a solid organ transplant: case report and review of the literature. Cutis 2008;81:127-30.

9. Batista MV, Pierrotti LC, Abdala E, et al. Endemic and opportunistic infections in Brazilian solid organ transplant recipients. Trop Med Int Health 2011;16:1134-42.

10. Ersan S, Celik A, Atila K, et al. Tuberculosis in renal transplant recipients. Renal Fail 2011;33:753-7.

11. Canet E, Dantal J, Blancho G, et al.
Tuberculosis following kidney transplantation: clinical features and outcome. A French multicentre experience in the last 20 years. Nephrol Dial Transplant 2011;26: 3773-8.

12. Lopez de Castilla D, Schluger NW. Tuberculosis following solid organ transplantation. Transpl Infect Dis 2010; 12:106-12. 